\title{
Dynamic Partitioning and Coverage Control with Asynchronous One-to-Base-Station Communication
}

\author{
Rushabh Patel, Paolo Frasca, Joseph W. Durham, Ruggero Carli, Francesco Bullo
}

\begin{abstract}
We propose algorithms to automatically deploy a group of mobile robots and provide coverage of a non-convex environment with communication limitations. In settings such as hilly terrain or for underwater ocean gliders, peer-to-peer communication can be impossible and frequent communication to a central base station may be impractical. This paper instead explores how to perform coverage control when each robot has only asynchronous and sporadic communication with a base station. The proposed algorithms rely upon overlapping territories, monotonically minimize suitable cost functions, and provably converge to a centroidal Voronoi partition. We also describe how the use of overlapping territories allows our algorithms to smoothly handle dynamic changes to the robot team.
\end{abstract}

\section{INTRODUCTION}

In applications such as environmental monitoring [1] or warehouse logistics [2] a team of robots is asked to perform tasks over a large space. The distributed environment partitioning problem consists of designing control and communication laws for individual robots such that the team divides a space into regions in order to optimize the quality of service provided. Coverage control additionally optimizes the positioning of robots inside of a region. Coverage control and territory partitioning have applications in many fields. In cyberphysical systems, applications include automated environmental monitoring [1], fetching and delivery [2], and other vehicle routing scenarios [3].

A broad discussion of partitioning and coverage control is presented in [4] which builds on the classic work of Lloyd [5] on algorithms for optimal quantizer design through "centering and partitioning." The Lloyd-type approach was first adapted for distributed coverage control

This work was supported in part by NSF grant CPS-1035917 and ARO grant W911NF-11-1-0092.

Rushabh Patel and Francesco Bullo are with the Department of Mechanical Engineering, University of California, Santa Barbara, CA, 93106 (r_patel, bullo) dengineering.ucsb.edu

Paolo Frasca is with the Department of Applied Mathematics, University of Twente, 7522 NB Enschede, The Netherlands p.frasca@utwente.nl

Joseph W. Durham is a Research Scientist at Kiva Systems, North Reading, MA joey@engineering.ucsb.edu

Ruggero Carli is with the Department of Information Engineering, University of Padova, Via Gradenigo 6/a, 35131 Padova, Italy carlirug@dei.unipd.it in [6] and has since seen many variations, including nonconvex environments [7], [8] and self-triggered coverage algorithms [9].

Many existing coverage control algorithms assume that robots can communicate peer-to-peer [6], but in some environments this is impractical. For example, underwater acoustic communication between ocean gliders is very low bandwidth and hilly or urban terrain can block radio communication. Instead, we present a coverage control algorithm for a team of robots which collectively maintain complete coverage of the environment and individually have only occasional contact with a central base station. This one-to-base-station communication model can represent ocean gliders surfacing to communicate with a tower [10], UAV data mules that periodically visit ground robots [11], or cost-mindful use of satellite or cellular communication. Our algorithm optimizes the response time of the team to service requests in a non-convex environment represented by a graph, with optimality defined by relevant "multicenter" cost functions for overlapping territories. Early work in coverage control of discrete non-convex domains (represented by graphs) is presented in [12]. Discrete coverage problems are closely related to the literature on data clustering and $k$-means [13], as well as the facility location or $k$-center problem [14].

There are several specific contributions of this paper. First, we present the first coverage control algorithm for an asynchronous one-to-base-station communication model. This model is realistic and relevant for a variety of application domains. We handle the time delay between when robots communicate with the base station using overlapping regions instead of a partition. The algorithm can be adapted for various cost-functions and also allows for heterogeneity among agents. Second, we prove that the algorithm converges to a centroidal Voronoi partition in finite time for two relevant costfunctions. Our Lyapunov argument is based on an adaptation of the standard partition-based coverage cost function. Third, we introduce the notion of Pareto-optimal partitions and provide a cost-function to achieve such a partition using our algorithm. Finally, we describe how the algorithm can seamlessly handle changes in the environment as well as unscheduled arrival, departure or 
change in functionality of robots from the team. This feature leverages overlapping regions, and also eases integration of coverage control with task servicing.

A preliminary version of this article has appeared at the 2011 IEEE Conference on Decision Control and European Control Conference as [15]. This article contains various results not found in [15]. First, we introduce a more general algorithm to achieve centroidal Voronoi partitions with one-to-base station communication architecture; we accomplish this by revising how updates occur in the algorithm. Second, we extend the framework of [15] by allowing for heterogeneous agents using weighted Voronoi partitions. Third, we introduce the notion of Pareto-optimal partitions and provide a method to converge to such a partition. Finally, simulation results have been extended and updated to demonstrate the performance of the new policies not present in [15].

\section{Preliminaries: Coverings of GraphS AND COST FUnCTIONS}

The one-to-base-station communication model studied in this paper requires that, in the design of coverage algorithms, we adopt overlapping coverings instead of partitions. In this Section we translate concepts used in partitioning of continuous environments [4] to coverings on graphs. In our notation, $\mathbb{R}_{>0}, \mathbb{R}_{\geq 0}$, and $\mathbb{Z}_{\geq 0}$ respectively denote the sets of positive, nonnegative and nonnegative integer numbers. Given a set $A,|A|$ denotes the number of elements in $A$. Given sets $A, B$, their union and intersections are denoted as $A \cup B$ and $A \cap B$, respectively, and their difference is $A \backslash B=\{a \in A \mid a \notin$ $B\}$.

\section{A. Graphs and Distances}

Let the finite set $Q$ be a set of points in a continuous environment. These points can represent locations or small areas of interest. They are assumed to be the nodes of an (undirected) weighted graph $G(Q)=(Q, E, l)$ with edge set $E \subset Q \times Q$ and weight map $l: E \rightarrow \mathbb{R}_{>0}$; we let $l_{e}>0$ be the weight of edge $e$. We assume that $G(Q)$ is connected and think of the edge weights as travel distances between nearby nodes.

In any weighted graph $G(Q)$ there is a standard notion of distance between vertices defined as follows. A path in $G$ is an ordered sequence of vertices such that any consecutive pair of vertices is an edge of $G$. The weight of a path is the sum of the weights of the edges in the path. Given vertices $h$ and $k$ in $G$, the distance between $h$ and $k$, denoted $d_{G}(h, k)$, is the weight of the lowest weight path between them, or $+\infty$ if there is no path. If $G$ is connected, then the distance between any two vertices is finite. By convention, $d_{G}(h, k)=0$ if $h=k$. Note that $d_{G}(h, k)=d_{G}(k, h)$, for any $h, k \in Q$.

\section{B. Coverings of Graphs}

We will be covering $Q$ with $n$ subsets or regions which will each be owned by an individual agent.

Definition 1 (n-Covering). Given the graph $G(Q)=$ $(Q, E, l)$, we define a $n$-covering of $Q$ as a collection $P=\left\{P_{i}\right\}_{i=1}^{n}$ of subsets of $Q$ such that:

(i) $\bigcup_{i=1}^{n} P_{i}=Q$;

(ii) $P_{i} \neq \emptyset$ for all $i \in\{1, \ldots, n\}$;

Let $\operatorname{Cov}_{n}(Q)$ to be the set of $n$-coverings of $Q$.

Note that a vertex in $Q$ may belong to multiple subsets in $P$, i.e., a vertex may be covered by multiple agents. The above definition is an important change from prior work [12].

We also have use for the concept of a partition of $Q$.

Definition 2 ( $n$-Partition). A $n$-partition is a n-covering with the additional property that:

(iii) if $i \neq j$, then $P_{i} \cap P_{j}=\emptyset$.

Let $\operatorname{Part}_{n}(Q)$ to be the set of $n$-partitions of $Q$.

Among the ways of covering $Q$, there is one which is worth special attention. Before we state the partition, let us define the vector of weights $w:=\left\{w_{1}, \ldots, w_{n}\right\}$, such that $w_{i}>0$ and $\sum_{j=1}^{n} w_{i}=1$. For brevity, we denote $\mathcal{W}=\left\{w \in \mathbb{R}_{>0}^{n} \mid \sum_{i=1}^{n} w_{i}=1\right\}$. Then given $w \in \mathcal{W}$ and a vector of distinct points $c \in Q^{n}$, the partition $P \in \operatorname{Part}_{n}(Q)$ is said to be a multiplicatively weighted Voronoi partition of $\mathrm{Q}$ generated by $c$ and weighted by $w$ if, for each $P_{i}$ and all $k \in P_{i}$, we have $c_{i} \in P_{i}$ and $\frac{1}{w_{i}} d_{G}\left(k, c_{i}\right) \leq \frac{1}{w_{j}} d_{G}\left(k, c_{j}\right)$, for $j \neq i$. The elements of $c$ are said to be the generators of the Voronoi partition multiplicatively weighted by $w$. Note that the multiplicatively-weighted Voronoi partition generated by $c$ and $w$ is not unique since how to assign tied vertices is unspecified. Multiplicatively-weighted Voronoi partition allow us to accommodate heterogeneous agents. For example, if agent $i$ is faster than another agent $j$ (i.e., $w_{i}>w_{j}$ ), it would make sense that agent $i$ should control more territory than agent $j$. Note that the vector of multiplicative weights, $w$, are not the same as the graph edge weights, $l$, defined in Section II-A From this point forward we refer to multiplicatively-weighted Voronoi partitions simply as Voronoi partitions and the vector of weights $w$ is given and fixed (unless we say otherwise). Given that weights are fixed, for the rest of the paper we refer to a Voronoi partition generated by $c$ and $w$ simply as a Voronoi partition generated by $c$.

\section{Cost Functions}

Let weight function $\phi: Q \rightarrow \mathbb{R}_{>0}$ be a bounded positive function which assigns a relative weight to each element of $Q$. The one-center function $\mathcal{H}_{1}$ gives the cost for a robot to cover a subset $A \subset Q$ from a vertex $h \in A$ 

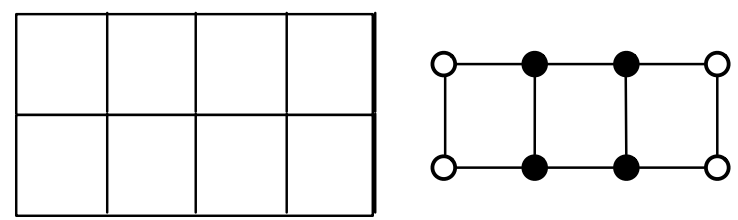

Fig. 1: The left image shows a grid environment whose corresponding graph representation is shown in the right image. Each cell in the grid represents a node in the graph and if two cells are adjacent, then there is an unit-weight edge between those nodes. The black nodes in the graph denote the set of generalized centroids for the corresponding grid environment.

with relative prioritization given by $\phi$ :

$$
\mathcal{H}_{1}(h ; A)=\sum_{k \in A} d_{G}(h, k) \phi(k) .
$$

A technical assumption is needed to define the generalized centroid of a subset. We assume from now on that a total order relation, $<$, is defined on $Q$ : hence, we can denote $Q=\{1, \ldots,|Q|\}$. With this assumption we can deterministically pick a centroid in $P_{i}$ which minimizes $\mathcal{H}_{1}$ as follows.

Definition 3 (Centroid). Let $Q$ be a totally ordered set, and let $A \subset Q$. We define the set of generalized centroids of $A$ as the set of vertices in $A$ which minimize $\mathcal{H}_{1}$, i.e.,

$$
\mathrm{C}(A):=\underset{h \in A}{\operatorname{argmin}} \mathcal{H}_{1}(h ; A) .
$$

In what follows, we drop the word "generalized" for brevity. Note that the centroid of a set always belongs to the set. Figure 1 shows an illustrative example of the set $\mathrm{C}(A)$ for a simple environment.

With these notions, we are ready to define the following useful cost functions. We can define the multi-center function $\mathcal{H}: Q^{n} \times \operatorname{Cov}_{n}(Q) \rightarrow \mathbb{R}_{\geq 0}$ to measure the cost for $n$ robots to cover a $n$-covering $P$ from the vertices $c \in Q^{n}$ with weights $w \in \mathcal{W}$ by

$$
\mathcal{H}(c, P)=\sum_{i=1}^{n} \sum_{k \in P_{i}} \frac{1}{w_{i}} d_{G}\left(c_{i}, k\right) \phi(k) .
$$

Note that if $w_{i}=w_{j}$ for all $i, j$, then the multicenter cost function above is the same as in [12]. We define the minimum cost-to-cover mapping $\mathcal{H}_{\min }: Q^{n} \times$ $\operatorname{Cov}_{n}(Q) \rightarrow \mathbb{R}_{\geq 0}$ by

$$
\mathcal{H}_{\text {min }}(c, P)=\sum_{k \in Q} \min _{i}\left\{\frac{1}{w_{i}} d_{G}\left(c_{i}, k\right) \mid k \in P_{i}\right\} \phi(k) \text {. }
$$

We aim to minimize these performance functions with respect to both the covering $P$ and the vertices $c$. In the motivational scenario we are considering, each robot will periodically be asked to perform a task somewhere in its region with tasks located according to distribution $\phi$. When idle, the robots would position themselves at the vertices $c$. By minimizing the coverage cost, the robot team minimizes the expected distance between a task and the furthest robot which can service the task.

We are almost ready to introduce a notion of optimal partition, the centroidal Voronoi partition. Our discussion begins with the following two results. The first one is a direct consequence of how we define a Voronoi partition and a centroid.

Proposition 4 (Properties of Multicenter Function). Let $P \in \operatorname{Part}_{n}(Q), c \in Q^{n}$ and $w \in \mathcal{W}$. If $P^{\prime}$ is a Voronoi partition generated by $c$, and $c^{\prime} \in Q^{n}$ satisfies $c_{i}^{\prime} \in$ $C\left(P_{i}\right)$ for all $i$, then

$$
\mathcal{H}\left(c, P^{\prime}\right) \leq \mathcal{H}(c, P) \text {, and } \mathcal{H}\left(c^{\prime}, P\right) \leq \mathcal{H}(c, P) \text {, }
$$

where the second inequality is strict if any $c_{i} \notin C\left(P_{i}\right)$.

Proposition 5 (Properties of $\mathcal{H}_{\text {min }}$ ). Let $P^{\prime} \in \operatorname{Part}_{n}(Q)$ be a Voronoi partition generated by $c \in Q^{n}$ (weighted by $w \in \mathcal{W}$ ) and let $P \in \operatorname{Cov}_{n}(Q)$ be any covering such that $P_{i}^{\prime} \subseteq P_{i}$ for all $i$. Let $\bar{P} \in \operatorname{Part}_{n}(Q)$ any partition satisfying $\bar{P}_{i} \cap P_{i}^{\prime} \ni c_{i}$ for all $i$. If $c^{\prime} \in Q^{n}$ satisfies $c_{i}^{\prime} \in C\left(P_{i}^{\prime}\right)$ for all $i$, then

$$
\begin{aligned}
\mathcal{H}_{\min }\left(c, P^{\prime}\right) & =\mathcal{H}_{\min }(c, P), \text { and } \\
\mathcal{H}_{\text {min }}\left(c, P^{\prime}\right) & \leq \mathcal{H}_{\min }(c, \bar{P}), \text { and } \\
\mathcal{H}_{\text {min }}\left(c^{\prime}, P^{\prime}\right) & \leq \mathcal{H}_{\min }\left(c, P^{\prime}\right),
\end{aligned}
$$

where the third inequality is strict if any $c_{i} \notin C\left(P_{i}\right)$.

Proof: Given that $P^{\prime} \subseteq P$ is a Voronoi partition generated by $c$, then given how a Voronoi partition and $\mathcal{H}_{\text {min }}$ are defined, the first statement holds. The second and third statements follow from Proposition 4 given that if $\bar{P}, P^{\prime}$ are partitions then $\mathcal{H}_{\min }(c, \bar{P})=\mathcal{H}(c, \bar{P})$ and $\mathcal{H}_{\text {min }}\left(c, P^{\prime}\right)=\mathcal{H}\left(c, P^{\prime}\right)$, respectively.

Propositions 4 and 5 imply that if $P \in \operatorname{Part}_{n}(Q)$ and $(c, P)$ minimizes $\mathcal{H}$ and $\mathcal{H}_{\text {min }}$, then $c_{i} \in C\left(P_{i}\right)$ for all $i$ and $P$ must be a Voronoi partition generated by $c$. This motivates the following definition.

Definition 6 (Centroidal Voronoi Partition). Given $w \in$ $\mathcal{W}, P \in \operatorname{Part}_{n}(Q)$ is a centroidal Voronoi partition of $Q$ if there exists a $c \in Q^{n}$ such that $P$ is a Voronoi partition generated by $c$ and $c_{i} \in \mathrm{C}\left(P_{i}\right)$ for all $i$.

For a given environment $Q$, a pair made of a centroidal Voronoi partition and the corresponding vector of centroids is locally optimal in the following sense: The cost functions $\mathcal{H}$ and $\mathcal{H}_{\text {min }}$ cannot be reduced by changing either $P$ or $c$ independently. Figure 2 demonstrates the difference between a Voronoi and centroidal Voronoi partition.

\section{Model, Problem, And Proposed Solution}

\section{A. One-to-Base-Station Robotic Network Model}

Given a team of $n$ robotic agents and a central base station, each agent $i \in\{1, \ldots, n\}$ is required to have the following basic computation capabilities: 

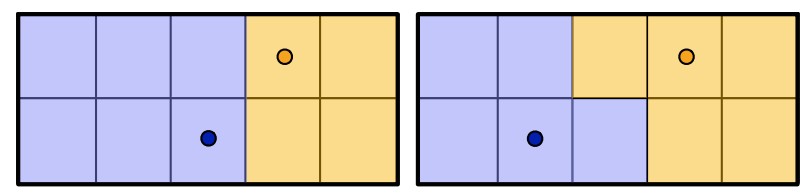

Fig. 2: The figure shows two environments with two agents. Each cell denotes a node in a graph and if two cells are adjacent, then there is an unit-weight edge between those nodes. The left image shows a Voronoi partition generated by the two agents. Note that the blue agent is not at its region's centroid. The right image is instead a centroidal Voronoi partition.

(C1) agent $i$ can identify itself to the base station; and

(C2) agent $i$ has a processor with the ability to store a region $S_{i} \subset G(Q)$ and a center $s_{i} \in S_{i}$.

Each $i \in\{1, \ldots, n\}$ is assumed to communicate with the base station according to the asynchronous one-tobase-station communication model described as follows:

(C3) there exists a finite upper bound $\Delta$ on the time between communications between $i$ and the base station. For simplicity, we assume no two agents communicate with the base station at the same time.

The base station must have the following capabilities:

(C4) it can store an arbitrary $n$-covering of $Q, P=$ $\left\{P_{i}\right\}_{i=1}^{n}$, a list of centroids $c \in Q^{n}$ and weights $w \in \mathcal{W}$

(C5) it can perform computations on subgraphs of $G(Q)$; and

(C6) it can store and operate on multiple $n$-coverings of $Q, P=\left\{P_{i}\right\}_{i=1}^{n}$ and a list of centroids $c \in Q^{n}$.

\section{B. Problem Statement}

Given weights $w \in \mathcal{W}$ assume that, for all $t \in \mathbb{R}_{\geq 0}$, each agent $i \in\{1, \ldots, n\}$ maintains in memory a subset $S_{i}(t)$ of environment $Q$ and a vertex $s_{i}(t) \in S_{i}(t)$. Our goal is to iteratively update the covering $S(t)=\left\{S_{i}\right\}_{i=1}^{n}$ and the centers $s(t)=\left\{s_{i}\right\}_{i=1}^{n}$ while solving the optimization problem:

$$
\min _{s \in Q^{n}} \min _{S \in \operatorname{Cov}_{n}(Q)} U(s, S),
$$

for some cost function $U(s, S)$ subject to the constraint that every node in the environment $Q$ maintains coverage from some agent, and subject to the constraint imposed by the robot network model with asynchronous one-tobase-station communication.

\section{The One-to-Base Coverage Algorithm}

Given the cost function defined by $U(s, S)$ and the One-to-Base Network model described by (C1)-(C6), we introduce the One-to-Base Coverage Algorithm to solve the optimization problem (1).

\section{One-to-Base Coverage Algorithm}

The base station maintains in memory an $n$-covering $P=\left\{P_{i}\right\}_{i=1}^{n}$, vector of locations $c=\left(c_{i}\right)_{i=1}^{n}$ and normalized weights $w=\left(w_{i}\right)_{i=1}^{n}$, while each robot $i$ maintains in memory a set $S_{i}$ and a vertex $s_{i}$. The base station maintains in temporary memory an $n$-coverings $\bar{P}=\left\{\bar{P}_{i}\right\}_{i=1}^{n}$ and $\overline{\bar{P}}=\left\{\bar{P}_{i}\right\}_{i=1}^{n}$, along with vectors $\bar{c}=\left(\bar{c}_{i}\right)_{i=1}^{n}$ and $\overline{\bar{c}}=\left(\overline{\bar{c}}_{i}\right)_{i=1}^{n}$ for computational purposes. At $t=0$, let $P(0) \in \operatorname{Cov}_{n}(Q), S(0)=P(0)$, and let all $c_{i}(0)$ 's be distinct. Assume that at time $t \in \mathbb{R}_{>0}$, robot $i$ communicates with the base station. Let $P^{+}, c^{+}, S_{i}^{+}$, and $s_{i}^{+}$be the values after communication. Then the base station executes the following actions:

1: update $\bar{P}:=P, \bar{c}:=c, \overline{\bar{P}}:=P, \overline{\bar{c}}:=c$,

2: compute sets

$$
\begin{aligned}
& P_{i,+}:=\left\{x \quad \in \quad Q \mid \frac{1}{w_{i}} d_{G}\left(x, c_{i}\right) \quad<\right. \\
& \left.\min \left\{\frac{1}{w_{j}} d_{G}\left(x, c_{j}\right) \mid x \in P_{j}, j \neq i\right\}\right\} \\
& P_{i,-}:=\left\{\begin{array}{l|l}
x \in P_{i} \cap\left(\cup_{i \neq j} P_{j}\right) \mid \frac{1}{w_{i}} d_{G}\left(x, c_{i}\right) \geq
\end{array}\right. \\
& \left.\min \left\{\frac{1}{w_{j}} d_{G}\left(x, c_{j}\right) \mid x \in P_{j}, j \neq i\right\}\right\} \\
& \text { 3: update } \overline{\bar{P}}_{i}:=\left(P_{i} \backslash P_{i,-}\right) \cup P_{i,+} \\
& \text { 4: for } k \in P_{i} \backslash c \text { do } \\
& \text { 5: } \quad \text { compute sets } \\
& P_{i,+}:=\left\{\begin{array}{lll|l}
x & \in & \frac{1}{w_{i}} d_{G}(x, k)<
\end{array}\right. \\
& \left.\min \left\{\frac{1}{w_{j}} d_{G}\left(x, c_{j}\right) \mid x \in P_{j}, j \neq i\right\}\right\} \\
& P_{i,-}:=\left\{x \in P_{i} \cap\left(\cup_{i \neq j} P_{j}\right) \mid \frac{1}{w_{i}} d_{G}(x, k) \geq\right. \\
& \left.\min \left\{\frac{1}{w_{j}} d_{G}\left(x, c_{j}\right) \mid x \in P_{j}, j \neq i\right\}\right\} \\
& \text { 6: } \quad \text { update } \bar{P}_{i}:=\left(P_{i} \backslash P_{i,-}\right) \cup P_{i,+} \\
& \text { 7: } \quad \text { update } \bar{c}_{i}:=k \\
& \text { 8: } \quad \text { if } U(\bar{c}, \bar{P})<U(\overline{\bar{c}}, \overline{\bar{P}}) \text { then } \\
& \text { 9: } \quad \text { update } \overline{\bar{P}}_{i}:=\bar{P}_{i} \\
& \text { 10: } \quad \quad \text { update } \overline{\bar{c}}_{i}:=\bar{c}_{i} \\
& \text { 11: } P_{i}^{+}:=\overline{\bar{P}}_{i} \\
& \text { 12: } c_{i}^{+}:=\overline{\bar{c}}_{i} \\
& \text { 13: tell agent } i \text { to set } S_{i}^{+}:=P_{i}^{+} \text {and } s_{i}^{+}=c_{i}^{+}
\end{aligned}
$$

Remark 7 (Constant cost). Given the constant cost function $U(c, P)=\alpha$ for $\alpha \in \mathbb{R}$, for a set of initial conditions $(c, P)$, the One-to-Base Coverage Algorithm produces a Voronoi partition generated by $c$.

Remark 8 (Full coverage). Notice that the set $P_{i,+}$ adds points to an agents environment from other agent's territory that are closer to it. Also, notice that $P_{i,-}$ only removes points from agent i's territory if another agent is covering that territory. Defining the sets in this way 
ensures that every point in the environment will always have coverage by some agent.

We have the following main result on the limit behavior of the algorithm.

Theorem 9 (Convergence of One-to-Base Coverage Algorithm $\left.\left(\mathcal{H}_{\min }\right)\right)$. Consider a network consisting of $n$ robots endowed with the computation capacities (C1), (C2) and communication capacity (C3), and a base station with capacities (C4), (C5) and (C6). Assume the network implements the One-to-Base Coverage Algorithm with $U(c, P)=\mathcal{H}_{\min }(c, P)$. Then the resulting evolution

$$
(s, S): \mathbb{R}_{\geq 0} \rightarrow Q^{n} \times \operatorname{Cov}_{n}(Q)
$$

converges in finite time to a pair $\left(s^{*}, S^{*}\right)$ composed of a centroidal Voronoi partition $S^{*}$ generated by $s^{*}$.

\section{Pareto-Optimal Partitions}

Using the algorithms described thus far, ties along partitions' boundaries are not handled in any optimal way and can often be improved. The major source of sub-optimal boundary allocation is due to the discrete nature of how centroids of a region are selected. Often times when an agent has more than one "center" location, the overall partition can become better balanced if the agent takes an alternate center value as its centroid. The following definition and proposition make this notion more precise.

Definition 10 (Pareto-Optimal Partition). Given a vector of positions $c=\left\{c_{1}, \ldots, c_{n}\right\}$ and a vector of weights $w=\left\{w_{1}, \ldots, w_{n}\right\}$, the (weighted) Voronoi partition $P$ generated by $c$ is Pareto-optimal if for all $\bar{c}=$ $\left\{c_{1}, \ldots, \bar{c}_{i}, \ldots, c_{n}\right\}$ for $i \in\{1, \ldots, n\}$ such that $\bar{c}_{i} \neq c_{i}$ and $\bar{c}_{i} \in P_{i}$, the Voronoi partition $\bar{P}$ generated by $(w, \bar{c})$ satisfies $\mathcal{H}(c, P) \leq \mathcal{H}(\bar{c}, \bar{P})$.

As an immediate consequence of the definition of a Pareto-optimal partition and of Proposition 4 we can conclude that every Pareto-optimal partition is also a centroidal Voronoi partition. However, the reverse implication does not hold, as shown in the following example.

Example 1 (One Dimensional Pareto-optimal Partition). Consider the three environments in Figure 3 each with two agents denoted by the colored circles. Assume that each cell denotes a node in the graph and that unitweight edges connect any adjacent cells. Assume $\phi$ is constant. If the environment is partitioned according to leftmost image of Figure 3, then each agent is at the centroid of its region, and the graph is a centroidal Voronoi partition whose multi-center function cost-tocover is $\mathcal{H}=4$. This partition is clearly not well balanced, and unless ties are broken in some non-trivial way, this is a valid (worst-case) partition that the system can reach. If however, the blue agent moves to its other centroid, as shown in the middle image, then the worst case partition must be a variant of the partition shown in the rightmost image whose cost-to-cover is $\mathcal{H}=3$. There exists no partition with smaller cost-to-cover (w.r.t. $\mathcal{H}$ ) by moving any single agent, and hence the partition in the rightmost image is Pareto-optimal.
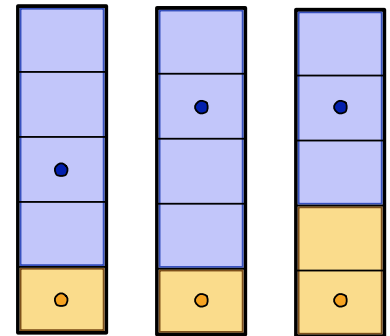

Fig. 3: The figure shows three environments with two agents. Each cell denotes a node in a graph, and if two cells are adjacent then there is an unit-weight edge between those nodes.

The above results give that Pareto-optimal partitions are a subset of centroidal Voronoi partitions. We can define the cost function, $U(c, P)$, in the One-to-Base Coverage Algorithm such that the algorithm converges to a Pareto-optimal partition. We define the new cost function

$$
\mathcal{H}_{\text {inf }}(c)=\sum_{k \in Q} \min _{i}\left\{\frac{1}{w_{i}} d_{G}\left(c_{i}, k\right) \mid k \in Q\right\} \phi(k) .
$$

Notice that this function is different from $\mathcal{H}_{\text {min }}$ in that it looks for the absolute minimum distance to a point, $k$. The function $\mathcal{H}_{\text {inf }}$ allows the case when $k \notin P_{i}$, but $\frac{1}{w_{i}} d_{G}\left(c_{i}, k\right)<\frac{1}{w_{j}} d_{G}\left(c_{j}, k\right)$, for all $j \neq i$. The $\mathcal{H}_{\text {inf }}$ function is linked to the multicenter function in the following sense.

Proposition 11 (Properties of $\mathcal{H}_{\text {inf }}$ ). Given $c \in Q^{n}$ and $w \in \mathcal{W}$, let $P$ be a Voronoi partition generated by $c$, then

$$
\mathcal{H}(c, P)=\mathcal{H}_{\text {inf }}(c) .
$$

Proof: Voronoi partitions are optimal in the sense that $\mathcal{H}(c, P)=\mathcal{H}_{\text {inf }}(c)$ by definition of $\mathcal{H}_{\text {inf }}$.

We are now ready to state the main result of this subsection. Given the One-to-Base Coverage Algorithm with $U(s, S)=\mathcal{H}_{\mathrm{inf}}(c)$ we have the following result.

Theorem 12 (Convergence of One-to-Base Coverage Algorithm $\left.\left(\mathcal{H}_{\mathrm{inf}}\right)\right)$. Consider a network consisting of $n$ robots endowed with the computation capacities (C1), (C2) and communication capacity (C3), and a base station with capacities (C4), (C5) and (C6). Assume the network implements the One-to-Base Coverage Algorithm with $U(c, P)=\mathcal{H}_{\mathrm{inf}}(c)$. Then the resulting evolution

$$
(s, S): \mathbb{R}_{\geq 0} \rightarrow Q^{n} \times \operatorname{Cov}_{n}(Q)
$$


converges in finite time to a pair $\left(s^{*}, S^{*}\right)$ composed of a Pareto-optimal partition $S^{*}$ generated by $s^{*}$.

Some remarks are in order. First, it is possible for the One-to-base Algorithm with $U=\mathcal{H}_{\min }$ to converge to a Pareto-optimal partition, however, it is not guaranteed as in the case with $U=\mathcal{H}_{\text {inf }}$. Second, if a partition is not Pareto-optimal then the cost to cover a region, in the context of the multi-center function, can be further decreased by making it Pareto-optimal. This point is clarified in Proposition 11, which relates the multicenter function to $\mathcal{H}_{\text {inf }}$. Finally, we emphasize that the difference in the cost-to-cover a region for a Paretooptimal partition versus a centroidal Voronoi partition decreases as the map defining a region becomes less coarse. This is because the notion of a centroidal Voronoi partition not being Pareto-optimal only exists when a region has more than one centroid, a property of discrete spaces but not of continuous ones. Therefore, as the grid approximating a region becomes less coarse, the more likely it is that a centroidal Voronoi partition is also Pareto-optimal.

\section{E. Combining $\mathcal{H}_{\mathrm{min}}$ and $\mathcal{H}_{\mathrm{inf}}$}

Although the One-to-Base Coverage Algorithm with $U=\mathcal{H}_{\text {inf }}$ is guaranteed to converge to a Pareto-optimal partition whereas the algorithm with $U=\mathcal{H}_{\text {min }}$ is not, the algorithm with $U=\mathcal{H}_{\text {min }}$ is still of practical importance. Computing $\mathcal{H}_{\text {inf }}$ requires that for each node in the graph, all agents compare their relative distances to that node regardless of whether that node exists in the agent's territory or not. The $\mathcal{H}_{\text {min }}$ function, however, only requires a comparison if the node belongs in the agent's territory. Given the computational capabilities of the base station being used, one method may be preferred over the other. A user can take advantage of both algorithm properties by running the algorithm with $U=\mathcal{H}_{\text {min }}$ until it converges to get an initial partition, and then run the algorithm with $U=\mathcal{H}_{\text {inf }}$ to reach a Pareto-optimal solution, if the solution system has not already reached it during the $U=\mathcal{H}_{\text {min }}$ portion of the algorithm. With a slight abuse of notation we will refer to this combined algorithm as the One-to-Base Coverage algorithm with $U=\mathcal{H}_{\text {min,inf }}$.

\section{IMPLEMENTATION AND SIMULATIONS}

In order to efficiently implement the One-to-Base Coverage Algorithm and under the assumption of using $\mathcal{H}_{\text {min }}$ or $\mathcal{H}_{\text {inf }}$ as cost functions, we provide the following revised version, which can be easily seen to be equivalent to that in Section III-C

\section{One-to-Base Coverage Algorithm - revised}

The base station maintains in memory an $n$-covering $P=\left\{P_{i}\right\}_{i=1}^{n}$, vector of locations $c=\left(c_{i}\right)_{i=1}^{n}$ and normalized weights $w=\left(w_{i}\right)_{i=1}^{n}$, while each robot $i$ maintains in memory a set $S_{i}$ and a vertex $s_{i}$. The base station maintains in temporary memory an $n$-covering $\bar{P}=\left\{\bar{P}_{i}\right\}_{i=1}^{n}$ and vectors $\bar{c}=\left(\bar{c}_{i}\right)_{i=1}^{n}$ and $\overline{\bar{c}}=\left(\overline{\bar{c}}_{i}\right)_{i=1}^{n}$ for computational purposes. At $t=0$, let $P(0) \in$ $\operatorname{Cov}_{n}(Q), S(0)=P(0)$, and let all $c_{i}(0)$ 's be distinct. Assume that at time $t \in \mathbb{R}_{>0}$, robot $i$ communicates with the base station. Let $P^{+}, c^{+}, S_{i}^{+}$, and $s_{i}^{+}$be the values after communication. Then the base station executes the following actions:

$$
\begin{aligned}
& \text { 1: update } \bar{P}:=P, \bar{c}:=c, \overline{\bar{c}}:=c, \bar{P}_{i}=Q \\
& \text { 2: for } k \in P_{i} \backslash c \text { do } \\
& \text { 3: } \quad \text { update } \bar{c}_{i}:=k \\
& \text { 4: } \quad \text { if } U(\bar{c}, \bar{P})<U(\overline{\bar{c}}, \bar{P}) \text { then } \\
& \text { 5: } \quad \text { update } \overline{\bar{c}}_{i}:=k \\
& \text { 6: } \text { compute sets } \\
& \quad P_{i,+}:=\left\{x \quad \in \quad Q \mid \frac{1}{w_{i}} d_{G}\left(x, \overline{\bar{c}}_{i}\right)<\right. \\
& \left.\quad \min \left\{\frac{1}{w_{j}} d_{G}\left(x, c_{j}\right) \mid x \in P_{j}, j \neq i\right\}\right\} \\
& \quad P_{i,-}:=\left\{x \in P_{i} \cap\left(\cup_{i \neq j} P_{j}\right) \mid \frac{1}{w_{i}} d_{G}\left(x, \overline{\bar{c}}_{i}\right) \geq\right. \\
& \left.\quad \min \left\{\frac{1}{w_{j}} d_{G}\left(x, c_{j}\right) \mid x \in P_{j}, j \neq i\right\}\right\} \\
& \text { 7: } P_{i}^{+}:=\left(P_{i} \backslash P_{i,-}\right) \cup P_{i,+} \\
& \text { 8: } c_{i}^{+}:=\overline{\bar{c}}_{i} \\
& \text { 9: } \text { tell agent } i \text { to set } S_{i}^{+}:=P_{i}^{+} \text {and } s_{i}^{+}=c_{i}^{+}
\end{aligned}
$$

This revised version of the algorithm takes advantage of how the cost functions $\mathcal{H}_{\text {min }}$ and $\mathcal{H}_{\text {inf }}$, and sets $P_{i,+}$ and $P_{i,-}$ are defined. Indeed, setting $\bar{P}_{i}=Q$ in line 1 avoids having to calculate $P_{i,+}$ and $P_{i,-}$ for every $k \in P_{i} \backslash c_{i}$, as was done in Section III-C because $\mathcal{H}_{\text {min }}$ and $\mathcal{H}_{\text {inf }}$ already distribute costs to nodes that are closer to one agent as opposed to another. Hence this implementation produces the same evolutions but requires less memory, as we no longer need the set $\overline{\bar{P}}$, and less computation time, as the sets $P_{i,+}$ and $P_{i,-}$ are calculated only once.

We are now ready to proceed with our simulation results, which are obtained by running the revised version of the algorithm. To demonstrate the utility of the Oneto-Base Coverage Algorithm for various values of cost function $U$, we implemented it using the open-source Player/Stage robot control system and the Boost Graph Library (BGL). All results presented here are generated using Player 2.1.1, Stage 2.1.1, and BGL 1.34.1. A nonconvex environment (borrowed from [12]) is specified with three robots. The free space is modeled using an occupancy grid with $0.6 \mathrm{~m}$ resolution, producing a lattice-like graph with all edge weights equal to $0.6 \mathrm{~m}$. The $0.6 \mathrm{~m}$ resolution is chosen so that each robot can fit in a grid cell.

One example with $U=\mathcal{H}_{\text {min,inf }}$ is shown in Figure 4 In the simulation, the robots have uniform weight assignment defined by $w_{i}=\frac{1}{3}$ for $i \in\{1, \ldots, 3\}$. We start with each robot owning the entire environment and 
stationed at its unique centroid as shown in the first panel, and then proceed by choosing a random robot to communicate with the base station at each iteration. The second panel shows an intermediate covering of the environment before convergence to a centroidal Voronoi partition. The third panel shows convergence of the $U=\mathcal{H}_{\text {min }}$ portion of the $U=\mathcal{H}_{\text {min,inf algorithm. The }}$ fourth panel shows the Pareto-optimal partition which is achieved after convergence of the $U=\mathcal{H}_{\text {inf }}$ portion of the $U=\mathcal{H}_{\text {min,inf }}$ algorithm. As can be seen, the movement of the robot relative to the third panel is marginal, but the partition appears to be more balanced and is still centroidal Voronoi. The cost to cover in terms of the multi-center function, $\mathcal{H}$, decreases from $\mathcal{H}=729$ to $\mathcal{H}=728$. Although the final partition and the decrease in cost-to-cover change only marginally in this example, the change can be much more noticeable as is explained in the following.

Another example with $U=\mathcal{H}_{\text {min,inf }}$ is shown in Figure 5. As before, the robot's have uniform weight assignment defined by $w_{i}=\frac{1}{3}$ for $i \in\{1, \ldots, 3\}$. The example starts with each agent owning the entire territory, the agents being stationed at their unique centroid, and the simulation continuing with the agents being selected at random to communicate with the base station. The second panel shows the convergence of the $U=\mathcal{H}_{\text {min }}$ portion of the algorithm, which leads to a final multi-center cost of $\mathcal{H}=804$. The third panel shows the update after the first iteration of the $U=\mathcal{H}_{\text {inf }}$ portion of the algorithm with the green agent. The update shows that the lower portion of the environment is getting less than optimal coverage and is improved by moving an agent closer to that region. The fourth panel shows the Pareto-optimal partition which is achieved after convergence of the $U=\mathcal{H}_{\text {inf }}$ portion of the $U=\mathcal{H}_{\text {min,inf }}$ algorithm. Notice that in this example, the final partition is quite different from the partition achieved at the end of the $U=\mathcal{H}_{\text {min }}$ portion of the algorithm. The final multi-center function cost of this partition is $\mathcal{H}=753$, which is a noticeable improvement in coverage.

Thus far we have looked at two representative examples of using the algorithm with $U=\mathcal{H}_{\text {min,inf }}$. These examples illustrate that, like centroidal Voronoi partitions, Pareto-optimal partitions are not necessarily unique, and that the evolution under the One-to-base station algorithm is only guaranteed to converge to a locally optimal solution. To see how the algorithm compares in general and for different choices of $U$, we simulate the algorithm with the same initial setup as shown in both Figure 4 and Figure 5. The One-to-Base Coverage Algorithm with $U=\mathcal{H}_{\text {min }}, U=\mathcal{H}_{\text {inf }}$, and $U=\mathcal{H}_{\min , \text { inf }}$ is run 100 times for each choice of $U$. Table II summarizes the final cost-of-coverage for each choice of $U$. We observe that the One-to-Base Coverage Algorithm with $U=\mathcal{H}_{\text {min }}$ converges to partitions that have the same minimum cost as those attained with the algorithm using $U=\mathcal{H}_{\text {inf }}$ or $U=\mathcal{H}_{\text {min,inf }}$. On the other hand, the maximum cost-to-cover with $U=\mathcal{H}_{\text {min }}$ can be much larger than with the other two choices of $U$. Of the three algorithms, the algorithm with $U=\mathcal{H}_{\text {inf }}$ converges consistently to partitions with the lowest coverage costs, however, as discussed earlier it is computationally the most expensive. Finally, the algorithm with $U=\mathcal{H}_{\text {min,inf }}$ behaves as expected, converging on average to partitions with values similar to that of the algorithm with $U=\mathcal{H}_{\text {inf }}$ although with a slightly larger deviation.

\begin{tabular}{ccccc}
\hline \hline Algorithm & Min & Mean & Max & StdDev \\
\hline $\mathcal{H}_{\text {min }}$ & 728 & 746.02 & 804 & 27.74 \\
$\mathcal{H}_{\text {inf }}$ & 728 & 730.26 & 732 & 1.92 \\
$\mathcal{H}_{\text {min,inf }}$ & 728 & 730.38 & 753 & 4.91 \\
\hline
\end{tabular}

TABLE I: Multi-center function cost-to-cover statistics for each algorithm from 100 simulation runs.

\section{A. Handling Dynamic Changes}

Evolving overlapping coverings in the One-to-Base Coverage Algorithm enables simple handling of environmental changes along with dynamic arrivals, departures, and even the disappearance of robots. Changes in the environment along with robot departures or disappearances can increase coverage cost, but those increases are only a transients and, with the appropriate algorithmic additions, the system will converge in finite steps after such an event. The One-to-Base Coverage Algorithm also has the added advantage that it can account for changes in robot performance due to changes in capability caused by potential damage to the hardware. The following algorithmic additions address how to handle the events described.

a) Environment Changes: Each region in the environment is initially assigned an importance according to the weight function $\phi(x)$. As robots explore the environment, they may determine that certain regions are more/less important than what was originally assigned. Robots can communicate this to the base station at which point the base station can update $\phi(x)$.

b) Arrival: When a new robot $i$ communicates with the base station, it can be assigned any initial $P_{i}$ desired. Possibilities include adding all vertices within a set distance of its initial position or assigning it just the single vertex which has the highest coverage cost in $Q$.

c) Departure \& Disappearance: A robot $i$ might announce to the base station that it is departing, perhaps to recharge its batteries or to perform some other task. In this situation, the base station can simply add $P_{i}$ to the territory of the next robot it talks to before executing the normal steps of the algorithm. The disappearance or failure of a robot $i$ can be detected if it does not 

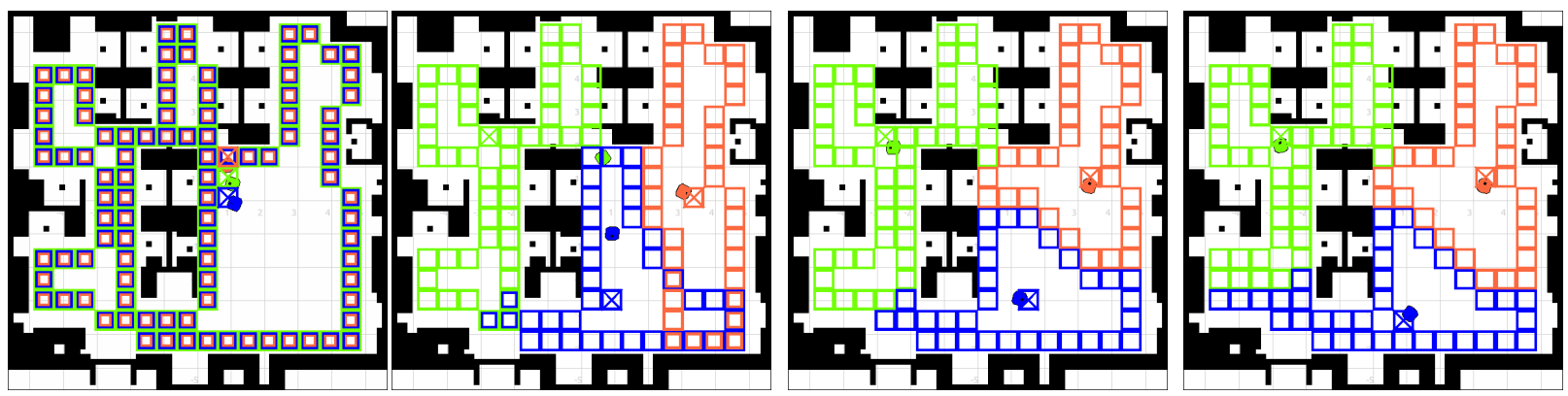

Fig. 4: Simulation of three robots partitioning an environment with black obstacles using the $\mathcal{H}_{\text {min,inf }}$ One-to-base station algorithm. The free space of the environment is modeled using the indicated occupancy grid where each cell is a vertex in the resulting graph. The robots' optimal coverage position is marked by an $\mathrm{X}$ and the boundary of each robot's territory drawn in its color. Some cells are on the boundary of multiple territories and for these we draw superimposed robot colors.
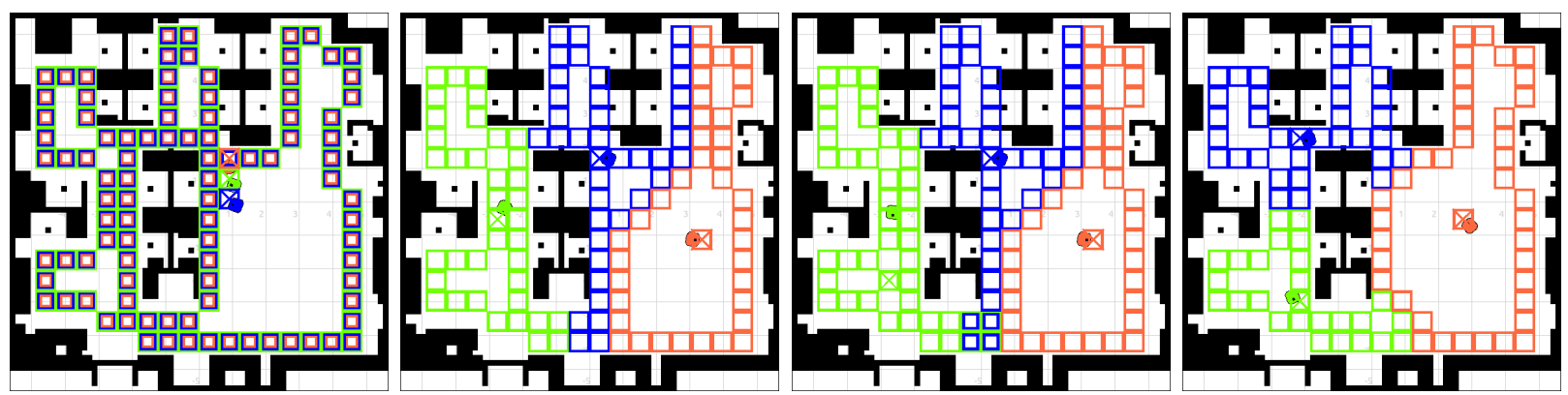

Fig. 5: Simulation of three robots partitioning an environment with black obstacles using the $\mathcal{H}_{\text {min,inf }}$ One-to-base station algorithm. The initial conditions are equivalent to that of Figure 4(d) however, note that the evolution of the algorithm is different.

communicate with the base station for longer than $\Delta$. If this occurs, then the departure procedure above can be triggered. Should $i$ reappear later, it can be handled as a new arrival or given its old territory.

d) Performance: Malfunction of a robot $i$ can be detected by the agent via self diagnosis and communicated to the base station. If this malfunction causes the robot to survey less territory, then $w_{i}$ will have changed, so the base station can simply re-normalize the vector of weights $w$.

\section{CONClusion}

We have described a coverage algorithm, with corresponding cost-functions, which uses the One-to-Base station communication architecture that drive territory ownership among a team of robots in a non-convex environment to a centroidal Voronoi partition in finite time. We have also defined the notion of Pareto-optimal partition and have provided a provably correct method to reach such a partition using the One-to-Base Coverage Algorithm. Finally, we have demonstrated the effectiveness of the algorithm through simulation, and have outlined various ways the algorithm can be adapted to allow for dynamic changes in the system. We have focused on dividing territory in this work, but the algorithm can easily be combined with methods to provide a service over $Q$, as in [16]. This work leaves various extensions open for further research. First, it would be worthwhile to adapt the algorithm to allow for areaconstrained partitions similar to the work done in [17], [18]. Second, we would like to extend the One-to-base Coverage Algorithm to other communication settings (e.g., directional or pair-wise gossip) to take advantage of the notion of Pareto-optimal partitions.

\section{APPENDIX}

In this appendix we prove Theorems 9 and 12 Any mention to the One-to-Base Coverage Algorithm in this appendix will refer to the version presented in Section III-C Their proof is based on the following convergence result for set-valued algorithms on finite state spaces, which can be recovered as a direct consequence of [19, Theorem 4.3].

Given a set $X$, a set-valued map $T: X \rightrightarrows X$ is a map which associates to an element $x \in X$ a subset $Z \subset X$. A set-valued map is non-empty if $T(x) \neq 0$ for all $x \in$ $X$. Given a non-empty set-valued map $T$, an evolution of the dynamical system associated to $T$ is a sequence 
$\left\{x_{n}\right\}_{n \in \mathbb{Z}_{>0}} \subset X$ with the property $x_{n+1} \in T\left(x_{n}\right)$ for all $n \in \mathbb{Z}_{\geq 0}$.

Lemma 13 (Convergence under persistent switches). Let $(X, d)$ be a finite metric space. Given a collection of maps $T_{1}, \ldots, T_{m}: X \rightarrow X$, define the set-valued map $T: X \rightrightarrows X$ by $T(x)=\left\{T_{1}(x), \ldots, T_{m}(x)\right\}$ and let $\left\{x_{n}\right\}_{n \in \mathbb{Z}_{\geq 0}}$ be an evolution of $T$. Assume that:

1) there exists a function $U: X \rightarrow \mathbb{R}$ such that $U\left(x^{\prime}\right)<U(x)$, for all $x \in X$ and $x^{\prime} \in T(x) \backslash\{x\}$; and

2) for all $i \in\{1, \ldots, m\}$, there exists an increasing sequence of times $\left\{n_{k} \mid k \in \mathbb{Z}_{\geq 0}\right\}$ such that $x_{n_{k}+1}=T_{i}\left(x_{n_{k}}\right)$ and $\left(n_{k+1}-n_{k}\right)$ is bounded.

Let $F_{i}=\left\{x \in X \mid T_{i}(x)=x\right\}$ be the set of fixed points of $T_{i}$. Then, for all $x_{0} \in X$ there exist $N \in \mathbb{N}$ and $\bar{x} \in\left(F_{1} \cap \cdots \cap F_{m}\right)$ such that $x_{n}=\bar{x}$ for all $n \geq N$.

Note that the existence of a common fixed point for the collection of maps $T_{i}$ is guaranteed by this result. We now apply Lemma 13 to the evolution of One-tobase Coverage Algorithm with $U(c, P)=\mathcal{H}_{\min }(c, P)$ and $U(c, P)=\mathcal{H}_{\text {inf }}(c)$, respectively. To do so, for each function given by $U(c, P)$, we must describe the algorithm as a set-valued map and find a corresponding Lyapunov function. The first step is possible because the One-to-Base Coverage Algorithm is well-posed in the sense of the following immediate result.

Proposition 14 (Well-posedness). Let $P \in \operatorname{Cov}_{n}(Q)$ and $c \in Q^{n}$ such that $c_{i} \in P_{i}$ and $c_{i} \neq c_{j}$ for all $i$ and all $j \neq i$. Then, $P^{+}$and $c^{+}$produced by the One-to-Base Coverage Algorithm meet the same criteria.

Given this result, the One-to-Base Coverage Algorithm can be written as a set valued map. For any $i \in$ $\{1, \ldots, n\}$, we define the map $T_{U, i}: Q^{n} \times \operatorname{Cov}_{n}(Q) \rightarrow$ $Q^{n} \times \operatorname{Cov}_{n}(Q)$ by

$$
\begin{aligned}
T_{U, i}(c, P)=\{ & \left\{c_{1}, \ldots, c_{i}^{+}, \ldots, c_{N}\right\}, \\
& \left.\left\{P_{1}, \ldots, P_{i}^{+}, \ldots, P_{n}\right\}\right\},
\end{aligned}
$$

where $c_{i}^{+}$and $P_{i}^{+}$are defined per the algorithm when $i$ is the communicating robot, and $U$ is dependent on the cost function we are referring to (i.e., $U=\mathcal{H}_{\text {min }}$ or $\left.U=\mathcal{H}_{\text {inf }}\right)$. Then we can define the set-valued map $T_{U}: Q^{n} \times \operatorname{Cov}_{n}(Q) \mapsto Q^{n} \times \operatorname{Cov}_{n}(Q)$ by

$$
T_{U}(c, P)=\left\{T_{U, 1}(c, P), \ldots, T_{U, N}(c, P)\right\} .
$$

Thus, the dynamical system defined by the application of the algorithm is described by $\left\{c^{+}, P^{+}\right\} \in T_{U}(c, P)$.

For our Lyapunov arguments we will need to define $M(P)$ as the set of vertices which are owned by multiple agents. We now proceed by stating two useful propositions, which allow us to conclude Theorem 9 .

Proposition 15 (Decaying $\mathcal{H}_{\text {min }}$ cost function). After each iteration of the one-to-base station algorithm if $\left(c^{+}, P^{+}\right) \neq(c, P)$ then one of the following holds: (i) $\mathcal{H}_{\min }\left(c^{+}, P^{+}\right)<\mathcal{H}_{\min }(c, P)$; or

(ii) $\mathcal{H}_{\min }\left(c^{+}, P^{+}\right)=\mathcal{H}_{\min }(c, P)$, and $\left|M\left(P^{+}\right)\right|<|M(P)|$.

Proof: If $c^{+}=c$ then $\mathcal{H}_{\min }\left(c^{+}, P^{+}\right) \leq$ $\mathcal{H}_{\text {min }}(c, P)$. This is a direct consequence of how the sets $P_{i,+}$ and $P_{i,-}$ are defined. Points are added to $P_{i}$ if and only if they are strictly closer to $c_{i}$ than any other center $c_{j}$ and hence the cost of $\mathcal{H}_{\min }$ must decrease by the addition of these points. Points are removed if and only if they are strictly farther away or tied points and so $\mathcal{H}_{\text {min }}$ must decrease or stay the same. If $c^{+} \neq c$ then by lines 8-10 of the algorithm $\mathcal{H}_{\min }\left(c^{+}, P^{+}\right)<\mathcal{H}_{\min }(c, P)$. For the case $\mathcal{H}_{\min }\left(c^{+}, P^{+}\right)=\mathcal{H}_{\min }(c, P)$ then for every $x \in P_{j} \backslash P_{i}$, for all $j \neq i$, there exists no point that is strictly closer to the center $c_{i}$ than any other center $c_{j}, j \neq i$. Therefore, no points can be added to $P_{i}^{+}$, and so if $P^{+} \neq P$ it must be the case that $\left|M\left(P^{+}\right)\right|<|M(P)|$.

Proposition 16 (Convergence of $T_{\mathcal{H}_{\text {min }}}$ ). The evolution of the One-to-Base Coverage Algorithm $(c(t), P(t))$ generated by the map $T_{\mathcal{H}_{\min }}$ converges in finite time to the intersection of the equilibria of the maps $T_{\mathcal{H}_{\min }, i}$, that is, to a pair $(c, P)$ where $P$ is a centroidal Voronoi partition generated by $c$.

Proof: The proof continues with the application of Lemma 13 to $(c(t), P(t))$. The algorithm is defined as the mapping $T_{\mathcal{H}_{\text {min }}}: Q^{n} \times \operatorname{Cov}_{n}(Q) \mapsto Q^{n} \times \operatorname{Cov}_{n}(Q)$ defined above, which is well-posed. We can form a Lyapunov function using Proposition 15 as follows. Since $Q$ is a finite set, there exists only a finite number of possible values for $\mathcal{H}_{\min }$ and $|M|$. Let $\epsilon_{m}$ be the magnitude of the smallest possible non-zero difference between two values of $\mathcal{H}_{\text {min }}$. Let $\alpha_{M}$ be larger than twice the maximum possible value of $|M|$. Consider the following function $V: Q^{n} \times \operatorname{Cov}_{n}(Q) \rightarrow \mathbb{R}_{\geq 0}$ :

$$
V(c, P)=\mathcal{H}_{\min }(c, P)+\frac{\epsilon_{m}}{\alpha_{M}}|M(P)| .
$$

With this scaling of $|M(P)|$ if $\mathcal{H}_{\text {min }}$ decreases $V$ necessarily decreases. Thus invoking Proposition 15. we conclude that if $\left(c^{\prime}, P^{\prime}\right) \in T_{\mathcal{H}_{\text {min }}}(c, P)$, then either $V\left(c^{\prime}, P^{\prime}\right)<V(c, P)$ or $\left(c^{\prime}, P^{\prime}\right)=(c, P)$. Thus, $V(c, P)$ fulfills assumption (ii). Finally the communication model (C3) assures that assumption (iii) is met. Then applying lemma 13, we are assured the dynamics converge to a fixed point $\left(c^{*}, P^{*}\right)$. It remains to show that $\left(c^{*}, P^{*}\right)$ is a centroidal Voronoi partition generated by $\left(w, c^{*}\right)$. If $P^{*}$ is not a partition, then $P_{i,-} \neq \emptyset$ and if the partition is not Voronoi then $P_{i,+} \neq \emptyset$. If $P^{*}$ is a Voronoi partition, generated by $\left(w, c^{*}\right)$ but $c_{i}^{*} \notin C\left(P_{i}^{*}\right)$ for any $i$, then from Proposition 5 and lines 8-10 of the algorithm, the cost to cover $P_{i}^{*}$ can be improved so $\left(c^{*}, P^{*}\right)$ cannot be a fixed point of $T_{\mathcal{H}_{\text {min }}}(c, P)$. Therefore, the fixed point is a centroidal Voronoi Partition. 
Since updates to agent $i$ in base-station memory also occur on the physical agent, we can conclude the convergence proof of Theorem 9 .

Finally, we state two propositions which allow us to conclude Theorem 12

Proposition 17 (Decaying $\mathcal{H}_{\mathrm{inf}}$ cost function). After each iteration of the one-to-base station algorithm if $\left(c^{+}, P^{+}\right) \neq(c, P)$ using $\mathcal{H}_{\text {inf }}$ as the cost function then one of the following holds:

(i) $\mathcal{H}_{\text {inf }}\left(c^{+}\right)<\mathcal{H}_{\text {inf }}(c)$; or

(ii) $\mathcal{H}_{\text {inf }}\left(c^{+}\right)=\mathcal{H}_{\text {inf }}(c)$, and

$\mathcal{H}_{\text {min }}\left(c^{+}, P^{+}\right)<\mathcal{H}_{\min }(c, P)$; or

(iii) $\mathcal{H}_{\text {inf }}\left(c^{+}\right)=\mathcal{H}_{\text {inf }}(c), \mathcal{H}_{\min }\left(c^{+}, P^{+}\right)=\mathcal{H}_{\min }(c, P)$ and $\left|M\left(P^{+}\right)\right|<|M(P)|$

Proof: If $c^{+}=c$ then $\mathcal{H}_{\min }\left(c^{+}, P^{+}\right) \leq \mathcal{H}_{\min }(c, P)$ and $\mathcal{H}_{\text {inf }}\left(c^{+}\right)=\mathcal{H}_{\text {inf }}(c)$. This is a direct consequence of how we define $\mathcal{H}_{\text {inf }}$ and the sets $P_{i,+}$ and $P_{i,-}$. Points are added to $P_{i}$ if and only if they are strictly closer to $c_{i}$ than any other center $c_{j}$ and hence the cost of $\mathcal{H}_{\text {min }}$ must decrease by the addition of these points. Points are removed if and only if they are strictly farther away or tied points and so $\mathcal{H}_{\text {min }}$ must decrease or stay the same. If $c^{+} \neq c$ then by the lines 8-10 of the algorithm $\mathcal{H}_{\mathrm{inf}}\left(c^{+}\right)<\mathcal{H}_{\mathrm{inf}}(c)$. For the case $\mathcal{H}_{\min }\left(c^{+}, P^{+}\right)=\mathcal{H}_{\min }(c, P)$ then for every $x \in P_{j} \backslash P_{i}$, for all $j \neq i$, there exist no point that is strictly closer to the center $c_{i}$ than any other center $c_{j}, j \neq i$. Therefore, no points can be added to $P_{i}^{+}$, and so if $P^{+} \neq P$ it must be the case that $\left|M\left(P^{+}\right)\right|<|M(P)|$.

Proposition 18 (Convergence of $T_{\mathcal{H}_{\text {inf }}}$ ). The evolution of the One-to-Base Coverage Algorithm $(c(t), P(t))$ generated by the map $T_{\mathcal{H}_{\mathrm{inf}}}$ converges in finite time to the intersection of the equilibria of the maps $T_{\mathcal{H}_{\mathrm{inf}}, i}$, that is, to a pair $(c, P)$ where $P$ is a Pareto-optimal partition generated by $c$.

Proof: The proof follows the lines of the proof of Proposition 16, with the important modification of using a different Lyapunov function, defined as follows. Let $\epsilon_{i}$ and $\epsilon_{m}$ be the magnitude of the smallest possible nonzero difference between two values of $\mathcal{H}_{\text {inf }}$ and $\mathcal{H}_{\text {min }}$, respectively. Let $\alpha_{m}$ and $\alpha_{M}$ be larger than twice the maximum possible value of $\mathcal{H}_{\text {min }}$ and $|M|$, respectively. If we define the function $V$ as

$$
V(c, P)=\mathcal{H}_{\text {inf }}(c)+\frac{\epsilon_{i}}{\alpha_{m}} \mathcal{H}_{\text {min }}(c, P)+\frac{\epsilon_{i} \epsilon_{m}}{\alpha_{m} \alpha_{M}}|M(P)|
$$

and we invoke Proposition 17 and Lemma 13. we conclude that the dynamics converge to a fixed point $\left(c^{*}, P^{*}\right)$.

It remains to show that $\left(c^{*}, P^{*}\right)$ is a Pareto-optimal partition generated by $c^{*}$. If $P^{*}$ is not a partition, then $P_{i,-} \neq 0$ and if the partition is not Voronoi then $P_{i,+} \neq 0$. Continuing by contradiction, assume that $c^{*}$ form a Voronoi partition which is not Pareto-optimal.
This implies that there exists a $c^{\prime} \in P$ where for at least one agent $c_{i}^{\prime} \neq c_{i}^{*}$ and a partition $P^{\prime}$ generated by $c^{\prime}$ such that $\mathcal{H}\left(c^{\prime}, P^{\prime}\right)<\mathcal{H}\left(c^{*}, P^{*}\right)$. By the definition of the algorithm and Proposition 11 this is not possible. Therefore the fixed point partition is Pareto-optimal.

\section{BIBLIOGRAPHY}

[1] E. Fiorelli, N. E. Leonard, P. Bhatta, D. A. Paley, R. Bachmayer, and D. M. Fratantoni. Multi-AUV control and adaptive sampling in Monterey Bay. IEEE Journal of Oceanic Engineering, 31(4):935-948, 2006.

[2] P. R. Wurman, R. D'Andrea, and M. Mountz. Coordinating hundreds of cooperative, autonomous vehicles in warehouses. AI Magazine, 29(1):9-20, 2008.

[3] B. Golden, S. Raghavan, and E. Wasil. The Vehicle Routing Problem: Latest Advances and New Challenges, volume 43 of Operations Research/Computer Science Interfaces. Springer, 2008.

[4] F. Bullo, J. Cortés, and S. Martínez. Distributed Control of Robotic Networks. Princeton University Press, 2009.

[5] S. P. Lloyd. Least squares quantization in PCM. IEEE Transactions on Information Theory, 28(2):129-137, 1982. Presented at the 1957 Institute for Mathematical Statistics Meeting.

[6] J. Cortés, S. Martínez, T. Karatas, and F. Bullo. Coverage control for mobile sensing networks. IEEE Transactions on Robotics and Automation, 20(2):243-255, 2004.

[7] L. C. A. Pimenta, V. Kumar, R. C. Mesquita, and G. A. S. Pereira. Sensing and coverage for a network of heterogeneous robots. In IEEE Conf. on Decision and Control, pages 3947-3952, Cancún, México, December 2008.

[8] S. Bhattacharya, R. Ghrist, and V. Kumar. Multi-robot coverage and exploration in non-Euclidean metric spaces. In Algorithmic Foundations of Robotics $X$, volume 86, pages 245-262. Springer, 2013.

[9] C. Nowzari and J. Cortés. Self-triggered coordination of robotic networks for optimal deployment. Automatica, 48(6):1077-1087, 2012.

[10] A. Pereira, H. Heidarsson, C. Oberg, D. Caron, B. Jones, and G. Sukhatme. A communication framework for cost-effective operation of AUVs in coastal regions. In A. Howard, K. Iagnemma, and A. Kelly, editors, Field and Service Robotics, volume 62 of Tracts in Advanced Robotics, pages 433-442. Springer, 2010.

[11] R. C. Shah, S. Roy, S. Jain, and W. Brunette. Data MULEs: modeling and analysis of a three-tier architecture for sparse sensor networks. Ad Hoc Networks, 1(2-3):215-233, 2003.

[12] J. W. Durham, R. Carli, P. Frasca, and F. Bullo. Discrete partitioning and coverage control for gossiping robots. IEEE Transactions on Robotics, 28(2):364-378, 2012.

[13] A. K. Jain, M. N. Murty, and P. J. Flynn. Data clustering: A review. ACM Computing Surveys, 31(3):264-323, 1999.

[14] V. V. Vazirani. Approximation Algorithms. Springer, 2001.

[15] J. W. Durham, R. Carli, P. Frasca, and F. Bullo. Dynamic partitioning and coverage control with asynchronous one-to-basestation communication. In IEEE Conf. on Decision and Control and European Control Conference, pages 5589-5594, Orlando, FL, USA, December 2011.

[16] F. Bullo, E. Frazzoli, M. Pavone, K. Savla, and S. L. Smith. Dynamic vehicle routing for robotic systems. Proceedings of the IEEE, 99(9):1482-1504, 2011.

[17] J. Cortés. Coverage optimization and spatial load balancing by robotic sensor networks. IEEE Transactions on Automatic Control, 55(3):749-754, 2010.

[18] R. Patel, P. Frasca, and F. Bullo. Centroidal area-constrained partitioning for robotic networks. ASME Journal of Dynamic Systems, Measurement, and Control, 136(3):031024, 2014.

[19] F. Bullo, R. Carli, and P. Frasca. Gossip coverage control for robotic networks: Dynamical systems on the space of partitions. SIAM Journal on Control and Optimization, 50(1):419-447, 2012. 